\section{Revista Brasileira de Tecnologia \\ Agroindustrial}

\title{
INFLUÊNCIA DA CEPA DE Saccharomyces cerevisiae NA CINÉTICA DE FERMENTAÇÃO DO VINHO DE MAÇÃ
}

\section{INFLUENCE OF THE STRAINS OF Saccharomyces cerevisiae IN THE KINETIC FERMENTATION OF THE APPLE WINE}

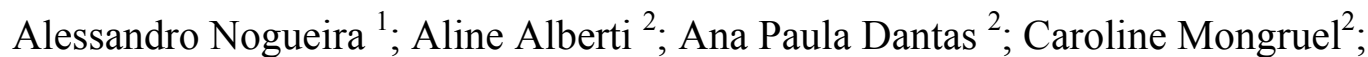 \\ Gilvan Wosiacki ${ }^{3}$ \\ 1Universidade Estadual de Ponta Grossa, UEPG, Ponta Grossa, Brasil. Bolsista PRODOC da CAPES. \\ E-mail: alessandro.nogueira@pesquisador.cnpq.br \\ 2Universidade Estadual de Ponta Grossa, UEPG, Ponta Grossa, Brasil. Bolsista de Iniciação Científica.
}

3Universidade Estadual de Ponta Grossa, UEPG, Ponta Grossa, Brasil. Bolsista de Produtividade em Pesquisa nível 1 CNPq. E-mail: gilvan.wosiacki@pesquisador.cnpq.br

\section{Resumo}

O produto da fermentação alcoólica do suco de maçã constitui a base para a elaboração da sidra, definida como uma bebida frisante de baixo grau alcoólico. A fermentação pode ocorrer de forma natural por uma microbiota complexa ou pelo inóculo de leveduras comerciais, selecionadas e melhoradas para a fabricação do vinho de uva. A avaliação de diferentes cepas de leveduras comerciais na fermentação da sidra torna-se importante a fim de que se possam definir parâmetros cinéticos e de qualidade sensorial. Neste trabalho o objetivo foi avaliar o comportamento fermentativo de três leveduras comerciais na elaboração do vinho de maçã. Foram utilizadas as cepas comerciais de Saccharomyces cerevisiae denominadas Uvaferm CK e Fermol®, e uma levedura de panificação. Houve diferença no crescimento máximo das três cepas sendo que a de

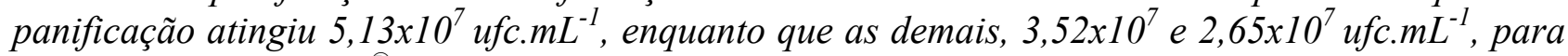
Uvaferm CK e Fermol ${ }^{\circledR}$, respectivamente. Houve diferença também na velocidade de consumo de de açúcares e na quantidade de nitrogênio consumida.
\end{abstract}

Palavras chaves: leveduras, vinho de maçã, fermentação.

\section{Introdução}

O processo de fermentação de suco de maçã visando à obtenção de bebida alcoólica já era conhecido no Mediterrâneo Oriental há mais de 2000 anos (LAPLACE et al., 2001) e é bem difundido o fato de que sucos de maçãs contêm os nutrientes necessários para o desenvolvimento de leveduras (SMOCK \& NEUBERT, 1950; BINNING \& POSSMANN, 1993; DOWNING, 1989). A principal fonte de carbono e energia são os açúcares, com teores acima de 100 g.L $\mathrm{L}^{-1}$ suficientes para

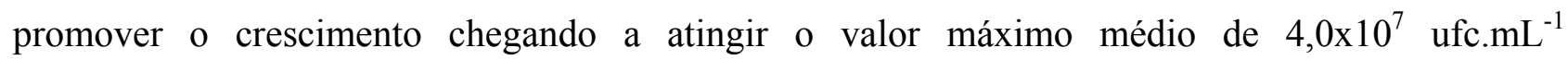
(NOGUEIRA \& WOSIACKI, 2005). 
Na fermentação, os compostos nitrogenados são utilizados pela Saccharomyces sp. durante a fase de crescimento, exercendo importantes funções na biossíntese de proteínas e nas funções enzimáticas, influenciando o crescimento e o metabolismo das leveduras, chegando a constituir 10\% da sua matéria seca (GOÑI e AZPILICUETA, 1999; JULIEN et al., 2000; JULIEN et al., 1994). Fatores como composição do mosto e cepa de levedura podem afetar a sua assimilação (JIRANEK et al., 1990; JULIEN et al., 2000; MANGINOT et al., 1998) assim como o seu metabolismo em leveduras pode conduzir a eficiência da fermentação alcoólica e afetar a qualidade do produto final (SALMON e. BARRE, 1998).

A quantidade de nitrogênio nos mostos de maçãs pode ser muito baixa $\left(<50 \mathrm{mg}\right.$. $\left.\mathrm{L}^{-1}\right)$ ou excessiva (>150 mg. $\left.\mathrm{L}^{-1}\right)$ em função, principalmente, da idade dos pomares e das adubações, respectivamente, o que torna os compostos nitrogenados ora limitantes do crescimento das leveduras, causando lentidão e/ou paradas de fermentação, ora causadores de instabilidades microbiológicas, como explosões de garrafas, no caso da sidra, gaseificadas naturalmente (NOGUEIRA \& WOSIACKI, 2005; PÉREZ-ZÚÑIGA et al., 1997). Além disso, o setor agroindustrial de processamento de fermentados de maçã, utiliza como prática corrente a adição de nutrientes (vitaminas e compostos nitrogenados) como forma de correção antes de inocular a levedura alcoólica. Este procedimento elimina o risco de lentidão ou paradas de fermentação, porém, como não é feita uma análise de nitrogênio antes desta correção, pode causar um aumento desnecessário do teor de nitrogênio, principalmente em situações onde o teor natural de compostos nitrogenados já está elevado.

Neste trabalho o objetivo foi avaliar a influência da cepa na cinética de fermentação alcoólica durante o processamento de vinhos de maçãs.

\section{Material e Métodos}

Amostras de $20 \mathrm{~kg}$ de maçãs do cultivar Fuji, adquiridas no comércio local foram utilizadas para elaboração dos vinhos. Os produtos químicos foram sempre de qualidade PA e as preparações enzimáticas da Novozymes do Brasil foram disponibilizadas pela LNF, de Bento Gonçalves, RS.

Processamento do suco. As maçãs selecionadas e lavadas foram trituradas em microprocessador (Processador Metvisa, Tipo MPA) e a massa obtida foi acondicionada em pacotes de plástico poroso que, superpostos, foram submetidos a uma pressão de $3 \mathrm{~kg} / \mathrm{cm}^{2}$ (Prensa hidráulica Eureka, Hoppe Ind. Ltda, Brasil) durante 5 minutos. O mosto obtido foi despectinizado mediante o uso de enzimas (Pectinex 3XL, Novozymes do Brasil), a uma proporção de $3 \mathrm{~mL} / \mathrm{hL}\left(60\right.$ minutos a $50^{\circ} \mathrm{C}$ ) e, após sedimentação a frio, foi trasfegado e engarrafado.

Processamento do vinho. O mosto clarificado foi acondicionado em dezesseis (16) erlenmeyers esterilizados munidos de batoque para estudos da cinética da fermentação. Em três conjuntos de 
cinco fermentadores foram inoculadas as leveduras Saccharomyces cerevisiae [1] Uvaferm CK Danstar Ferment GAC; [2] Fermol ${ }^{\circledR}$ e [3] levedura de panificação, todas com uma população

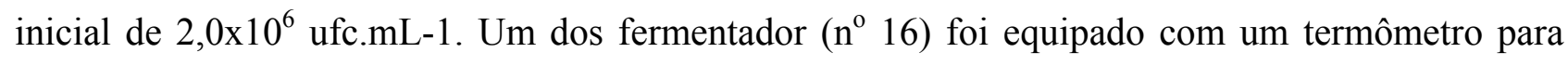
monitoramento da temperatura. A cinética de fermentação foi calculada pelo monitoramento da perda de massa dos fermentadores (BELY et al., 1990; ROGER et al., 2002) durante aproximadamente 15 dias (tempo de fermentação). O monitoramento da fermentação transcorreu nos tempos zero, 12, 24, 36, 120 e 384 horas. Ao final de cada tempo, o mosto em fermentação foi centrifugado a $2000 \mathrm{rpm}$ (Centrifuga Fanem Ltda, Modelo 214) durante 20 minutos sendo em seguida trasfegado, engarrafado e armazenado em baixa temperatura.

Análises. Para a contagem de unidades formadoras de colônias (UFC) de leveduras foi utilizada câmera de Neubauer (XB-K-25, SMIC, China) de acordo com os procedimentos de LEE et al.,(1981). Os teores de etanol foram determinados por ebuliometria. Os teores de açúcares redutores foram determinados pelo método de Somogyi-Nelson, bem como os açúcares redutores totais, após a hidrólise da sacarose com HCL $6 \mathrm{~N}\left(67^{\circ} \mathrm{C}\right.$ durante 5 minutos). A glucose foi determinada pelo método enzimático da glucose oxidase acoplada a peroxidase. A sacarose foi calculada através da diferença entre açúcares redutores totais e açúcares redutores, e a frutose com a diferença entre estes e a glucose. Todos os açúcares foram expressos em g.100mL-1. A acidez titulável total foi determinada pela neutralização com $\mathrm{NaOH} 0,1 \mathrm{~N}$ até $\mathrm{pH} 8,33$ com fenolftaleína e calculada como ácido málico expresso em g.100mL $\mathrm{mL}^{-1}$ (TANNER \& BRUNNER, 1985). O nitrogênio total foi quantificado por neutralização com $\mathrm{HCl} 0,02 \mathrm{~N}$, com solução de ácido bórico ( $2 \%$ mais indicadores), após ter sido destilado em destilador de nitrogênio com $\mathrm{NaOH} 50 \%$, sendo expresso em mg.100mL-1 (BARON et al., 1977). Os compostos fenólicos totais foram quantificados com o reativo de Folin-Ciocalteau utilizando-se a catequina como padrão para a determinação colorimétrica (RSK, 1987).

\section{Resultados e Discussão}

O tempo de 15 dias de fermentação na obtenção de vinho de maçã com as três diferentes cepas em temperatura entre 18 e $25^{\circ} \mathrm{C}$ foi suficiente para a exaustão dos açúcares fermentescíveis e produção de etanol ao redor de 7,0 $0^{\circ}$. Os resíduos de açúcares totais, observado na Tabela 1 correspondem aos carboidratos de baixa fermentabilidade como a xilose, galactose, rafinose $\mathrm{r} o$ poliol dorbitol, os quais permanecem no vinho de maçã (FULEKI et al., 1994).

Os teores de ácido málico aumentaram de $0,37 \mathrm{~g} \cdot 100 \mathrm{~mL}^{-1}$ para valores entre 0,44 a 0,46 g. $100 \mathrm{~mL}^{-1}$ devido à liberação de ácidos orgânicos durante o metabolismo da levedura (JONES et al., 1981). 
O teor de nitrogênio do mosto $\left(161,3 \mathrm{mg} . \mathrm{L}^{-1}\right)$ é considerado elevado, não sendo totalmente consumido pela levedura. A levedura de panificação apresentou um maior consumo de nitrogênio (96 mg. $\left.\mathrm{L}^{-1}\right)$ seguida da cepa Fermol $\left(93 \mathrm{mg} \cdot \mathrm{L}^{-1}\right)$ porém a cepa Uvaferm consumiu apenas 79 mg.L $\mathrm{L}^{-1}$. Esses valores refletem a quantidade de nitrogênio necessário para a formação de biomassa, que pode ser maior em função de carências nutricionais como oxigênio e fitosteróis utilizados na síntese do ergosterol da membrana plasmática, vitaminas como a tiamina e aminoácidos assimiláveis. Esses compostos podem ser considerados como os fatores de crescimento da levedura em anaerobiose na fermentação do mosto de maçã (NOGUEIRA, 2003).

A diminuição dos fenóis está relacionada com associações com a parede celular da levedura e perdas na filtração (papel filtro) por fixação na fibra celulose (RENAR et al., 2001) o que implica na diminuição da cor do produto final em comparação ao mosto despectinizado.

Na Figura 1 podem ser observadas as curvas de crescimento de cada levedura e que a cepa de panificação apresentou um crescimento mais rápido em relação às demais. A levedura Uvaferm apresentou a menor biomassa. As diferenças de crescimento refletiram em diferenças de velocidade de consumo dos açúcares, ou seja, a levedura de panificação apresentou uma maior população e consequentemente um consumo mais rápido desses carboidratos.

Tabela 1- Parâmetros físico-químicos do vinho de maçã.

\begin{tabular}{|c|c|c|c|c|}
\hline \multirow{2}{*}{$\begin{array}{l}\text { Parâmetros } \\
\text { físico-químicos }\end{array}$} & \multirow{2}{*}{$\begin{array}{l}\text { Composição do } \\
\text { mosto de maçã }\end{array}$} & \multicolumn{3}{|c|}{ Composição do vinho de maçã } \\
\hline & & Fermol $^{\circledR}$ & Panificação & Uvaferm CK \\
\hline Açúcares totais, g. $100 \mathrm{~mL}^{-1}$ & $12,22 \pm 0,58$ & $0,14 \pm 0,01$ & $0,13 \pm 0,02$ & $0,15 \pm 0,01$ \\
\hline Ácido málico, g. $100 \mathrm{~mL}^{-1}$ & $0,37 \pm 0,04$ & $0,45 \pm 0,01$ & $0,46 \pm 0,01$ & $0,44 \pm 0,00$ \\
\hline Nitrogênio total, mg. $\mathrm{L}^{-1}$ & $161,3 \pm 2,82$ & $68,14 \pm 0,98$ & $64,95 \pm 1,59$ & $82,09 \pm 0,23$ \\
\hline Fenóis totais, $\mathrm{mg} . \mathrm{L}^{-1}$ & $321,71 \pm 34,54$ & $266,13 \pm 19,58$ & $277,64 \pm 34,10$ & $298,26 \pm 26,22$ \\
\hline Etanol, ${ }^{\circ} \mathrm{GL}$ & 0,0 & $7,17 \pm 0,25$ & $7,42 \pm 0,11$ & $7,42 \pm 0,11$ \\
\hline
\end{tabular}

Ao analisar a cinética de fermentação observa-se um tempo de adequação, ao meio, maior para a levedura Uvaferm, fato este que pode ser observado na curva de crescimento, no tempo da velocidade máxima (1,2 dias), que foi o dobro do tempo demandado pelas outras duas cepas e na aceleração máxima que foi baixa $\left(3,76 \mathrm{~g} \cdot \mathrm{L}^{-1} \cdot \mathrm{d}^{-2}\right)$ sendo que para as leveduras de Panificação e Fermol os valores foram superiores 8,33 e $5,44 \mathrm{~g} . \mathrm{L}^{-1} \cdot \mathrm{d}^{-2}$, respectivamente (Tabela 2). 
Tabela 2- Parâmetros cinéticos da fermentação de vinho de maçã.

\begin{tabular}{lccc}
\hline Parâmetros fermentativos & Fermol $^{\circledR}$ & Panificação & Uvaferm CK \\
\hline Velocidade máxima, g. $\mathrm{L}^{-1} \cdot \mathrm{d}^{-1}$ & 4,42 & 5,30 & 4,87 \\
Momento de Vmáx., d & 0,60 & 0,60 & 1,20 \\
Aceleração máxima, g.L $\mathrm{L}^{-1} \cdot \mathrm{d}^{-2}$ & 5,44 & 8,33 & 3,76 \\
Momento de Amáx., d & 0,80 & 0,60 & 1,20 \\
População inicial, ufc.mL & $2,00 \times 10^{6}$ & $2,00 \times 10^{6}$ & $2,00 \times 10^{6}$ \\
População máxima, ufc.mL & $2,-1$ & $5,13 \times 10^{7}$ & $3,52 \times 10^{7}$ \\
Perda de gás carbônico, g. $\mathrm{L}^{-1}$ & 29,67 & 28,34 & 29,70 \\
\hline
\end{tabular}

Na Figura 1 podem ser observadas as curvas de cinéticas calculadas a partir da perda de peso dos fermentadores pela liberação do gás carbônico durante a fermentação alcoólica, das três leveduras em estudo. A velocidade de fermentação alcançou valores nulos em 13, 11 e 14 dias para as leveduras Fermol, Panificação e Uvaferm, respectivamente, sendo estes valores indicadores do final dos açúcares nos mostos.

Entretanto, o vinho de maçã obtido pela levedura de Panificação apresentou aromas de fermento os quais não têm boa aceitação, porém o vinho de maçã fermentado pela cepa Uvaferm, que apresentou fermentação mais lenta foi o que apresentou o melhor aroma, com notas de "frutado" (dados não apresentados).

Figura 1- Parâmetros cinéticos de fermentação do vinho de maçã produzido com as cepas: Curva de crescimento e consumo de açúcares (A), Fermol $^{\circledR}$ (B), Uvaferm (C) e Panificação (D).

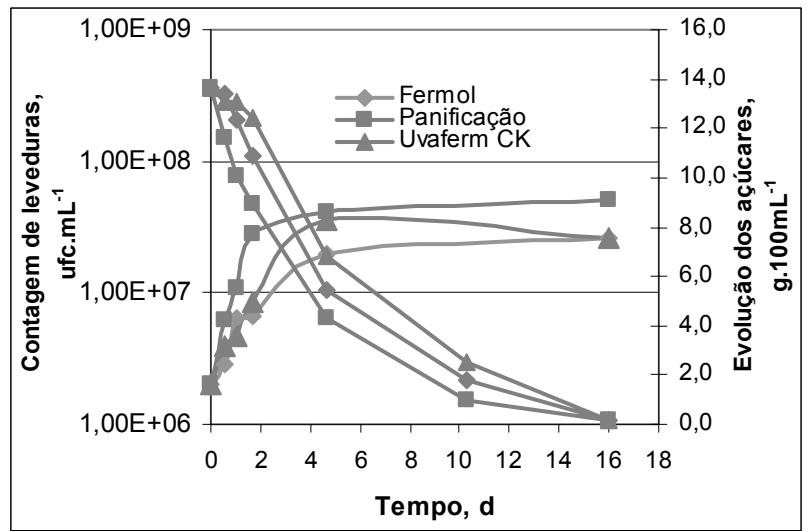

(A)

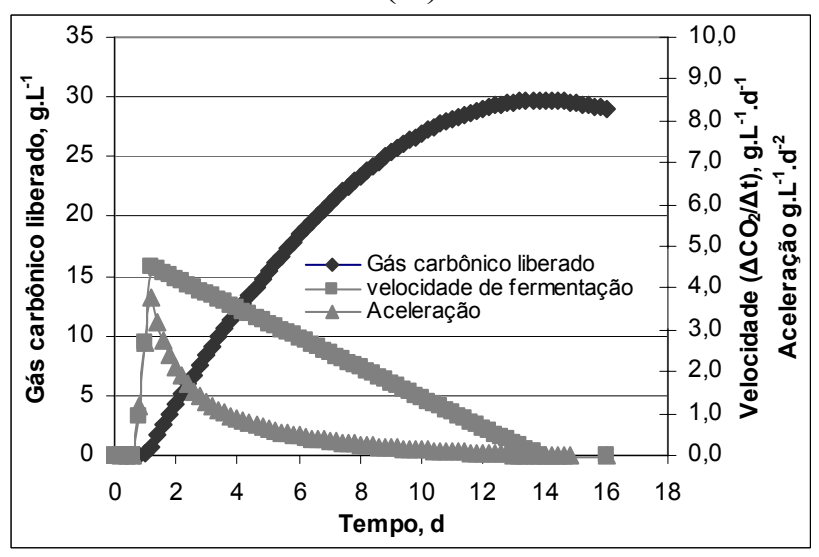

(C)

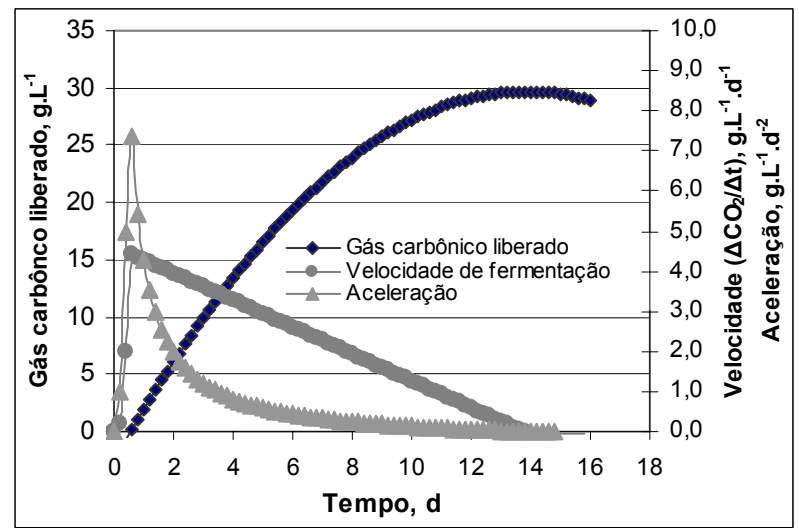

(B)

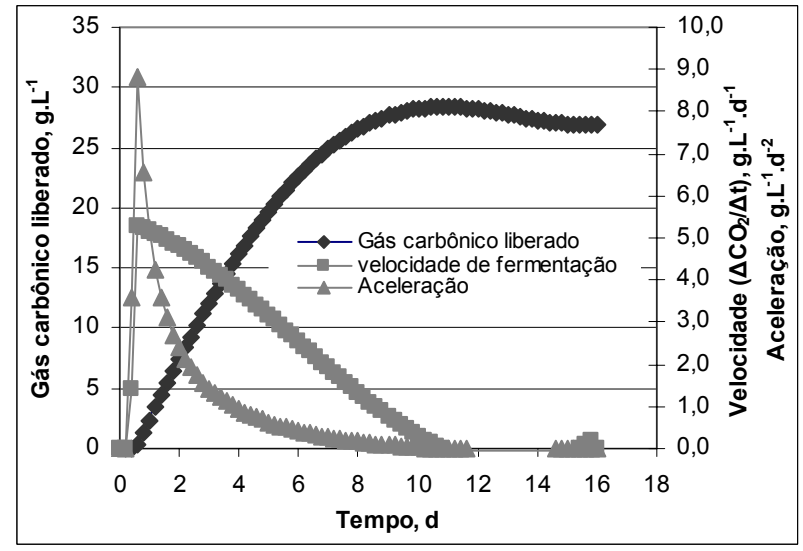

(D) 


\title{
4. Conclusão
}

Os resultados demonstraram as características de fermentação de cada cepa, sendo que as leveduras Saccharomyces cerevisiae em estudo apresentaram diferenças de adaptação ao meio, de população máxima, consumo de nitrogênio e velocidade de fermentação.

Em função da cepa utilizada como agente de fermentação a velocidade e término do processo e, em conseqüência, a qualidade de seus produtos, serão diferentes e isto deve ser de conhecimento do setor agroindustrial de processamento de maçã.

\begin{abstract}
The product of alcoholic fermentation of the apple juice constitutes the base for the elaboration of the cider-apple, defined as a sparkling drink of low alcoholic degree. The fermentation can occur in the presence of natural or commercial yeast, usually selected and improved for the manufacture of grape wine. The evaluation of different strains of commercial yeast in the apple cider fermentation becomes important to define kinetic parameters and sensorial aspects. This work was done to evaluate the fermentative behavior of three commercial yeasts in apple wine processing. Two strains of Saccharomyces cerevisiae (Uvaferm CK; Fermol ${ }^{\circledR}$ ) and baker yeast were used. It was found difference concerning maximum growth, baker yeast reaching $5,13 \times 10^{7} \mathrm{ufc} . \mathrm{mL}^{-1}$, Uvaferm $3,52 \times 10^{7}$ and Fermol, 2,65 $\times 10^{7}$ ufc. $\mathrm{mL}^{-1}$. It was found differences concerning substrate consumption what should be observed during the processing in this condition.
\end{abstract}

Keymords: yeast, apple wine, fermentation.

\section{Referências}

BINNIG, R.; POSSMANN, P. Apple Juice. In: NAGY, S.; CHEN, C. S.; SHAW, P. E. Fruit Juice. Processing Technology. Auburndale: Agscience, 1993, p. 271-317.

DOWNING, D. L. Apple cider. In: DOWNING, D. L. Processed apple products. 1989. p. 169187.

GOÑI, D. T.; AZPILICUETA, C. A. Use of nitrogen compounds in spontaneous and inoculated wine fermentations. Journal of Agricultural and Food Chemistry, v. 47, n. 10, 1999, p. 40184024.

IAL. Métodos físicos e químicos para análise de alimentos. In: IAL. Normas Analíticas. São Paulo: Instituto Adolfo Lutz, 3 ed., 1976.

JIRANEK, M., LANGRIDGE, P., HENSCHKE, P.A. Nitrogen requeriments of yeast during wine fermentation. Proceedings of the $7^{\circ}$ Australian Wine Industry Technical Conference. Adelide. Australian Industrial Publishers S.A. 1990. p. 166-177.

JONES, R. P.; PAMMENT, N.; GREENFIELD, P. F. Alcohol fermentation by yeasts. The effect of environmental and other variables. Process Biochemistry, n. 1981, p. $42-49$.

JULIEN, A.; ROUSTAN, J. L.; DULAU, L.; SABLAYROLLES, J.M. Comparison of nitrogen and oxygen demands of enological yeasts: technological consequences. American Journal of Enology and Viticulture, v. 51, n. 3, 2000, p. 215-222.

LAPLACE, J. M.; JACQUET, A.; TRAVERS, I.; SIMON, J. P.; AUFFRAY, Y. Incidence of land and physicochemical composition of apples on the qualitative and quantitative development of 
microbial flora during cider fermentations. Journal of the Institute of Brewing, v. 107, n. 4, 2001, p. 227-233.

LEE, S. S.; ROBINSON, F. M.; WANG, H. Y. Rapid determination of yeast viability. Biotechnology and Bioengineering Symposium, n. 11, 1981, p. 641-649.

NOGUEIRA, A.; WOSIACKI, G. Sidra. In: VENTURINI FILHO, W. G. Tecnologia de bebidas: matéria-prima, processamento, BPF/APPCC, legislação, mercado. Botucatu: Edgard Blüchen, 2005, p. 383-422.

NOGUEIRA, A. Tecnologia de processamento sidrícola. Efeitos do oxigênio e do nitrogênio na fermentação lenta da sidra. Tecnologia de processamento sidrícola. Efeitos do oxigênio e do nitrogênio na fermentação lenta da sidra. 210. Doutorado em Processos Biotecnológicos Agroindustriais. Universidade Federal do Paraná. Curitiba. 2003.

PÉREZ-ZÚÑIGA, F. J.; ABAD, F. B.; CARTAGENA, L. G. Residual proteins and amino nitrogen in fermented wine and beer: must variety and yeast interactions. $Z$ Lebensm Unters Forsch $A, v$. 205, n. 1997, p. 165-169.RSK. RSK- Wert. Die Gesamtdarstellung. Bonn: Flüssiges Obst GmbH, 1987, p. 204.

SALMON, J. M.; BARRE, P. Improvement of nitrogen assimilation and fermentation kinetics under enological conditions by derepression of alternative nitrogen-assimilatory pathways in an industrial Saccharomyces cerevisiae strain. Applied and Environmental Microbiology, v. 64, n. 10, 1998, p. 3831-3837.

RENARD, C. M. G. C.; BARON, A.; GUYOT, S.; DRILLEAU, J. F. Interactions between apple cell walls and native apple polyphenols: quantification and some consequences. International Journal of Biological Macromolecules, v. 29, n. 2, 2001, p. 115-125.

SMOCK, R. M.; NEUBERT, A. M. Apples and apples products. New York. Interscience Publishers. 1950. 2 ed. p. 486.

TANNER, H., BRUNNER, H.R. Getränke Anlytik - Untersuchungsmethode für dia Laborund Betriebspraxis. Wädesnwill: Verlag Helles, 1985, p. 206.

\section{Primeiro autor:}

Nome completo: Alessandro Nogueira

Filiação institucional: Universidade Estadual de Ponta Grossa

Função ou cargo ocupado: Bolsista de Pós-doutoramento (PRODOC/CAPES)

Endereço completo para correspondência (bairro, cidade, estado, país e CEP): Departamento de Engenharia de Alimentos, Av. Carlos Cavalcanti, n.4748, CEP 8030-900, Campus de Uvaranas, Ponta Grossa, PR.

Telefones para contato: 4232203093

e-mail: alessandro.nogueira@pesquisador.cnpq.br 